\title{
Human equilibrative nucleoside transporter 1 is associated with the chemosensitivity of gemcitabine in human pancreatic adenocarcinoma and biliary tract carcinoma cells
}

\author{
RYUTARO MORI $^{1,2}$, TAKASHI ISHIKAWA ${ }^{1}$, YASUSHI ICHIKAWA ${ }^{1}$, KOICHI TANIGUCHI ${ }^{1}$, \\ RYUSEI MATSUYAMA ${ }^{1}$, MICHIO UEDA ${ }^{1}$, YOSHIRO FUJII ${ }^{1}$, ITARU ENDO ${ }^{1}$, \\ SHINJI TOGO ${ }^{1}$, PETER V. DANENBERG ${ }^{2}$ and HIROSHI SHIMADA ${ }^{1}$ \\ ${ }^{1}$ Department of Gastroenterological Surgery, Yokohama City University Graduate School of Medicine, \\ 3-9 Fukuura, Kanazawa-ku, Yokohama 236-0004, Japan; ${ }^{2}$ University of Southern California/Norris \\ Comprehensive Cancer Center, NOR 5318, 1441 East Lake Avenue, Los Angeles, CA 90033, USA
}

Received January 8, 2007; Accepted February 14, 2007

\begin{abstract}
Gemcitabine has been one of the most commonly used agents for pancreatic adenocarcinoma chemotherapy, but the determinants of the sensitivity of and resistance to this agent are not yet fully understood. In this study with pancreatic carcinoma and biliary tract carcinoma cell lines, we examined the gene expression levels of nucleotide transporters and others related to the metabolism of gemcitabine in the light of sensitivity to this agent. Quantitative RT-PCR demonstrated that one of the nucleotide transporter genes; human equilibrative nucleoside transporter 1 (hENT1) was associated with the sensitivity to gemcitabine as represented by $\mathrm{IC}_{50}$, while the other genes for nucleotide transporter and metabolism were not. We conclude that increased hENT1 expression is a most important determinant of gemcitabine sensitivity at least in an in vitro study.
\end{abstract}

\section{Introduction}

The results of the treatment of patients with pancreatic adenocarcinoma are mostly miserable because of the advancement of the disease and its resistance to chemotherapy. Gemcitabine (2',2'-difluorodeoxycytidine) is one of the most commonly used chemotherapeutic agents in pancreatic adenocarcinoma and

Correspondence to: Dr Ryutaro Mori, 1640 Marengo st. 6th floor, Los Angeles, CA 90033, USA

E-mail: rmori@ck2.so-net.ne.jp

Abbreviations: hENT1, human equilibrative nucleoside transporter 1; hCNT1 and 3, human concentrative nucleoside transporter 1 and 3; dCK, deoxycytidine kinase; CDA, cytidine deaminase; RR, ribonucleotide reductase; RT-PCR, reverse transcriptional polymerase chain reaction; $\mathrm{IC}_{50}$, inhibitory concentration $50 \%$

Key words: hENT1, gemcitabine, pancreatic adenocarcinoma, biliary tract carcinoma biliary tract carcinoma, and has been shown to have marginal effects on the clinical outcome (1). This agent, like other nucleoside-derived drugs, must first be transported into the cell and then be metabolized to exert its clinical action. The transport of gemcitabine occurs via the nucleoside transporters, and in particular, human equilibrative nucleoside transporter 1 (hENT1), and concentrative nucleoside transporter 1 and 3 (hCNT1 and 3) play a central role in transporting gemcitabine into pancreatic adenocarcinoma cells. Once inside the cell, gemcitabine is phosphorylated to its active metabolites, $\mathrm{dFdCTP}$, and it is mainly incorporated into DNA leading to masked chain termination $(2,3)$. In addition, the active metabolites can inhibit ribonucleotide reductase (RR), enhancing the gemcitabine activation, leading to cell death (4). In this process, a potent inhibitor of ribonucleotide reductase (RR) is the most important gene. The initial phosphorylation step also requires the presence of deoxycytidine kinase $(\mathrm{dCK})$, which is known as the rate-limiting enzyme in gemcitabine activation, and cytidine deaminase (CDA) catalyzes the deamination of gemcitabine (4).

There are several reports regarding the mechanism of chemosensitivity to gemcitabine in lung, ovarian and bladder cancer. These suggested that a deficiency in deoxycytidine kinase, increased deamination and ribonucleotide reductase and decreased influx into the cell may contribute to impaired drug responsiveness (5-7).

To our knowledge, however, there is no comprehensive study of these genes in pancreatic adenocarcinoma cell lines in respect to gemcitabine sensitivity. In this study, we have examined the association between expression levels of all these genes and sensitivity to gemcitabine. With a panel of cell lines derived from human pancreatic adenocarcinoma, the mRNA expression levels were quantitated with real-time PCR, and gemcitabine chemosensitivity was examined with a cell inhibition study.

\section{Materials and methods}

Cell lines. The following three human pancreatic adenocarcinoma cell lines, a gall bladder carcinoma cell line, and a bile duct carcinoma cell line were used in this study: 


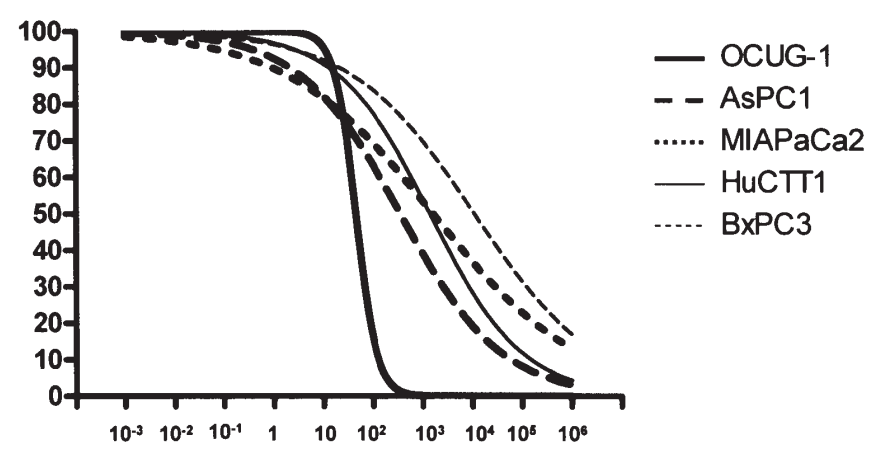

Figure 1. Chemosensitivity assay.

respectively, MIAPaCa2, AsPC1, BxPC3, OCUG-1 and HuCTT1 from the Health Science Research Resource Bank.

MIAPaCa2 was grown in MEM, AsPC1, BxPC3 and HuCTT1 were grown in RPMI-1640, and OCUG-1 was grown in DMEM, all supplemented with $10 \%$ FBS. Cells were maintained as monolayer cultures at $37^{\circ} \mathrm{C}$ in a $5 \% \mathrm{CO}_{2}$ atmosphere and subcultured every 3-4 days. Cells were counted routinely using trypan blue stain, $0.4 \%$ (Gibco) and a BurkerTurk deep counter, except for the drug sensitivity assay.

Chemicals. Gemcitabine was a gift from Eli Lilly Pharmaceuticals (Indianapolis, IN).

Total-RNA isolation and cDNA synthesis. Total-RNA was isolated from the cells with RNeasy mini kits (Qiagen, Valencia, CA) containing DNase-treatment, and cDNA was then synthesized using $\mathrm{TaqMan}^{\circledR}$ reverse transcription reagents (Applied Biosystems) according to the manufacturer's specifications.

Quantitative RT-PCR. The primer sets for RT-PCR of the hENT1, dCK, CDA, RRM1 and RRM2 amplification were as follows: hENT1, 5'-caggcaaagaggaatctgga-3' and 5'-ggcccaac cagtcaaagata-3'; dCK, 5'-gctgcagggaagtcaacatt-3' and 5'-cattc agagaggcaagctga-3'; CDA, 5'-aagtcagcctactgccctac-3' and 5'-tcaggctggagtgtaatctgg-3'; RRM1, 5'-gccaataaagatcgcctgaa-3' and 5'-ttgttcccaccttgatccac-3'; and RRM2, 5'-ggctggctgtgactta ccat-3' and 5'-actcagagggggcagagaat-3'; GAPDH. The primers were designed using published sequence data from GenBank and a BLAST search (http://www..rcbi.nlm.nih.ga/BLAST/).

The LightCycler FastStart DNA Master SYBR-Green I kit (Roche Applied Science, New Zealand) was used as the basis for the reaction mixture, using a $20-\mu 1$ volume in each reaction capillary. The final reaction mix included a dNTP mix (with dUTP instead of dTTP), SYBR-Green I dye, the hot-start enzyme FastStart Taq DNA polymerase, 2-5 mM
$\mathrm{MgCl}_{2}$ and $0.5 \mu \mathrm{M}$ of each primer. A negative control with PCR-grade water rather than template DNA was always used with the samples. Cycle conditions were optimized regarding the annealing temperature and holding times. Amplification conditions involved a pre-incubation at $95^{\circ} \mathrm{C}$ for $10 \mathrm{~min}$ followed by amplification of the target DNA for 45 cycles $\left(95^{\circ} \mathrm{C}\right.$ for $10 \mathrm{sec}, 60^{\circ} \mathrm{C}$ for $10 \mathrm{sec}$ and $72^{\circ} \mathrm{C}$ for $\left.20 \mathrm{sec}\right)$ with a transition rate of $20^{\circ} \mathrm{C} / \mathrm{sec}$. Melting curve analysis was performed at a linear temperature transition rate of $0.1{ }^{\circ} \mathrm{C} / \mathrm{sec}$ from 65 to $95^{\circ} \mathrm{C}$ with continuous fluorescence acquisition. This step was followed by a cooling step at $4^{\circ} \mathrm{C}$ for $30 \mathrm{sec}$. The fluorescence detected in channel F1 was analyzed by the LightCycler analysis software at the end of the run. The crossing points (beginning of the PCR exponential phase) for each reaction were determined by the Second Derivative Maximum algorithm. Each expression level was relative to that of GAPDH.

Chemosensitivity assay. Cells were cultured at 5000 cells/ well in 96-well tissue culture plates. To assess cell viability, stepwise 10 -fold dilutions of the anticancer drug were added $2 \mathrm{~h}$ after plating, and the cultures were incubated at $37^{\circ} \mathrm{C}$ for $72 \mathrm{~h}$ at the end of the culture period, $20 \mu 1$ of [3-(4,5-dimethylthiazol-2-yl)-5-(3-carboxymethoxyphenyl)-2-(4-sulfophenyl)2H-tetrazolium, inner salt] solution (CellTiter 96 ${ }^{\circledR}$ AQueous One Solution Cell Proliferation Assay; Promega, Madison, WI) was added, then the cells were incubated for a further $4 \mathrm{~h}$ and the absorbance was measured at $490 \mathrm{~nm}$ using an Immuno Reader NJ-2001 (Inter Med). Mean values were calculated from three independent experiments carried out in quadruplicate.

Statistical analysis. Spearman's test was used for analysis of the correlation between nucleoside transporter or metabolic enzyme gene and $\mathrm{IC}_{50}$ values for gemcitabine. The level of significance was set at 5\%, using a two-sided analysis.

\section{Results}

Chemosensitivity in pancreatic adenocarcinoma and bile duct carcinoma cell lines. In the 5 cell lines, sensitivities to gemcitabine widely varied from $1.1 \times 10^{4}$ to $6.8 \times 10^{1}$ of $\mathrm{IC}_{50}$. OCUG-1 was the most sensitive to gemcitabine in these lines, and BxPC3 was the least (Fig. 1 and Table I).

Expression of hENT1 and relationship to the cytotoxicity of gemcitabine. Quantitative RT-PCR revealed different expression mRNA levels of hENT1, dCK, CDA, RRM1 and RRM2 in the 5 cell lines (Figs. 2 and 3). Among them, hENT1 showed a variation of expression levels with the highest in OCUG-1 and the lowest in BxPC3.

Table I. $\mathrm{IC}_{50}$ values in pancreatic adenocarcinoma and biliary tract carcinoma cell lines.

\begin{tabular}{llllll}
\hline & OCUG-1 & AsPC1 & MIAPaCa2 & HuCTT1 & BxPC3 \\
\hline Gemcitabine $\mathrm{IC}_{50}(\mathrm{nM})$ & $6.8 \times 10^{1}$ & $3.5 \times 10^{2}$ & $1.6 \times 10^{3}$ & $1.4 \times 10^{3}$ & $1.1 \times 10^{4}$ \\
\hline
\end{tabular}




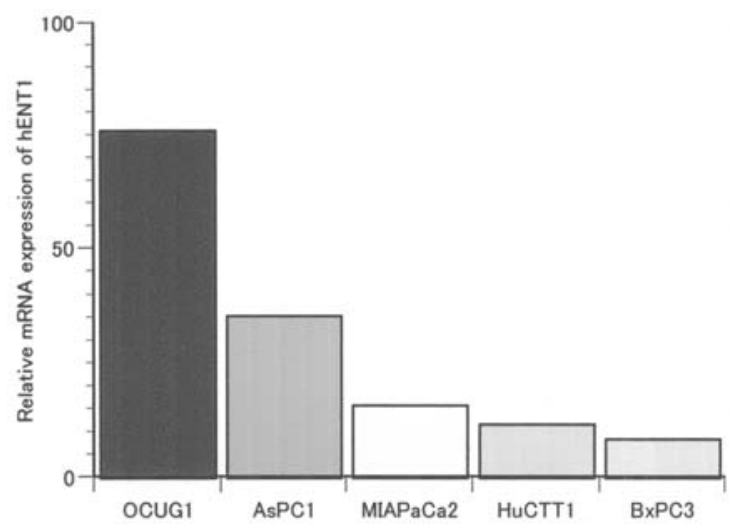

Figure 2. Qualitative RT-PCR analysis of hENT1 expression in pancreatic adenocarcinoma and biliary tract carcinoma cell lines. Total-RNA was extracted and processed as described in Materials and methods.

Relationship between chemosensitivity to gemcitabine and expression of $d C K, C D A, R R M 1$ and RRM2. The $\mathrm{IC}_{50}$ values of cell lines to gemcitabine were compared to the mRNA expression levels of hENT1, dCK, CDA, RRM1 and RRM2. Among them, hENT1 expression levels were significantly correlated with the $\mathrm{IC}_{50}$ values for gemcitabine in these cell lines ( $r=-0.900, P=0.037$ ) (Fig. 4). However, no significant correlation to $\mathrm{IC}_{50}$ was found in the other genes of DCK, CDA, RRM1 (Figs. 5 and 6).

\section{Discussion}

Gemcitabine is one of the most promising anti-metabolic agents against cancer. Among this type of agents, 5-fluorouracil (5-FU) is the most intensively investigated drugs in terms of

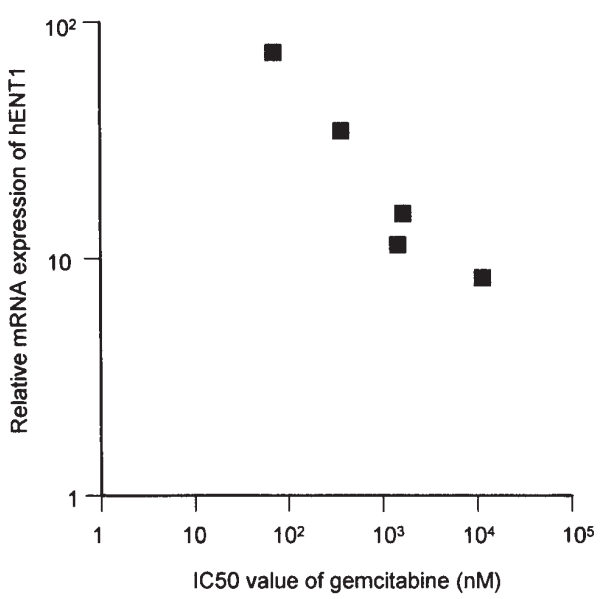

Figure 4. Relationship between gemcitabine sensitivity and basal expression level of hENT1 in pancreatic adenocarcinoma and biliary tract carcinoma cell lines. There was a clear correlation between the levels of hENT1 expression and the $\mathrm{IC}_{50}$ values. All $\mathrm{IC}_{50}$ values are the mean of the values in three independent sensitivity tests performed in quadruplicate. Expression levels are relative to the expression of GAPDH. Statistical significance of the correlations was determined by means of Spearman's correlation test.

the mechanism of its effects. Based on many basic and clinical studies on 5-FU, thymidylate synthase (TS); a target enzyme for 5-FU and dihydropyrimidine dehydrogenase (DPD), the degrading enzyme of 5-FU, have been recently utilized on a clinical basis, not only for modifying the efficacy of 5-FU, but also for predicting its clinical effect. These studies on 5-FU could be a good paradigm for investigating the sensitivity and resistance mechanisms of other anti-metabolic agents such as gemcitabine. Theoretically, dCK and CDA for gemcitabine could be equivalent to TS and DPD for 5-FU, respectively.
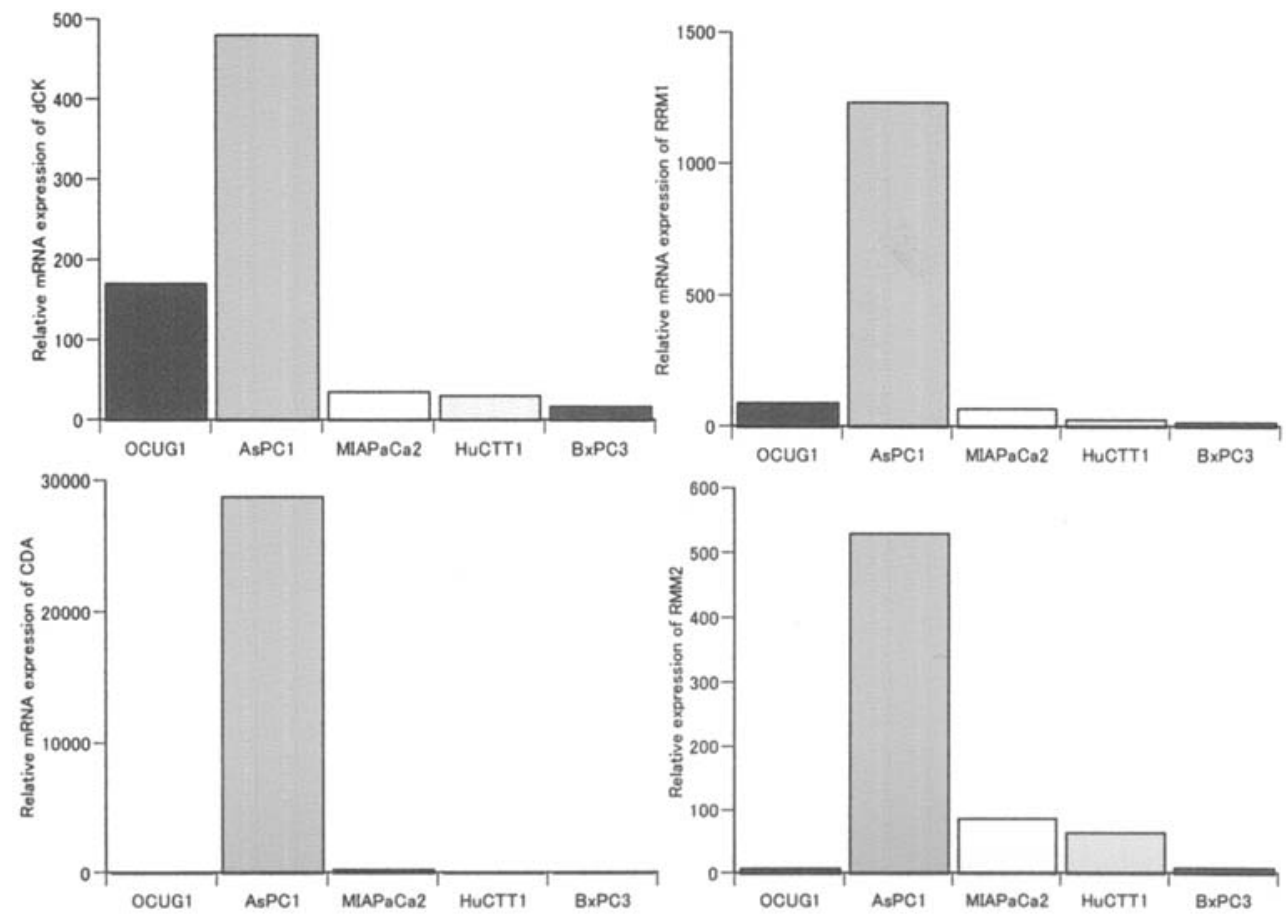

Figure 3. Qualitative RT-PCR analysis of the expression levels of dCK, CDA, RRM1 and RRM2 in pancreatic adenocarcinoma and biliary tract carcinoma cell lines. 

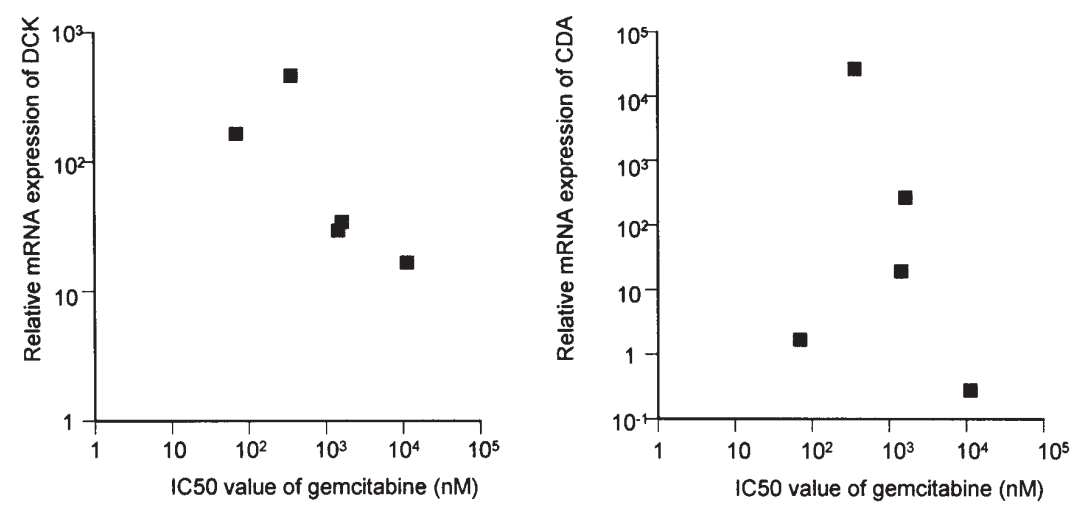

Figure 5. Relationship between gemcitabine sensitivity and the basal expression level of dCK and CDA in pancreatic adenocarcinoma and biliary tract carcinoma cell lines. There was no correlation between the levels of dCK or CDA expression and the $\mathrm{IC}_{50}$ values.
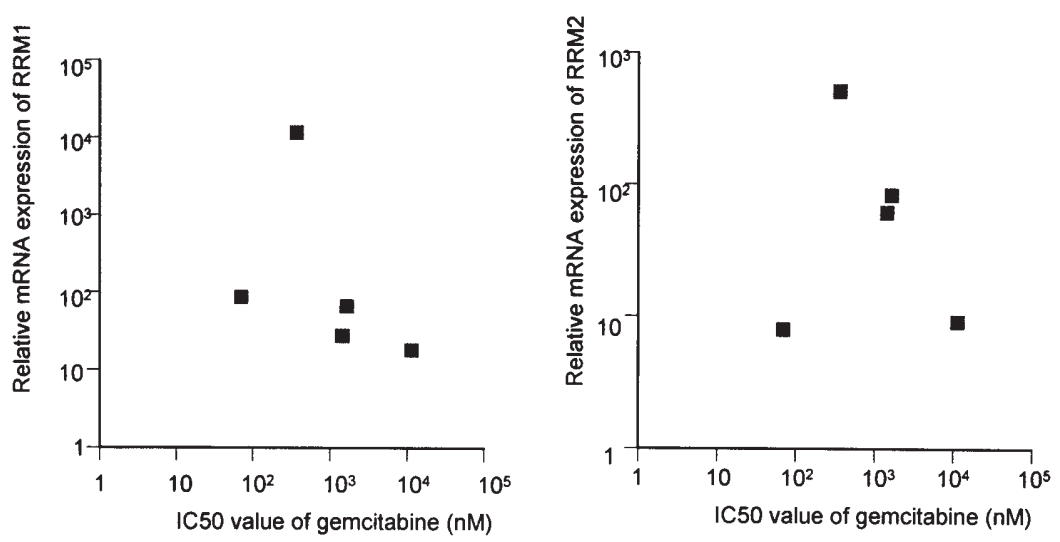

Figure 6. Relationship between gemcitabine sensitivity and the basal expression level of RRM1 and RRM2 in pancreatic adenocarcinoma and biliary tract carcinoma cell lines. There was no correlation between the levels of RRM1 or RRM2 expression and the $\mathrm{IC}_{50}$ values.

Thus far, dCK enzyme activity is reported to be associated with gemcitabine sensitivity in both in vitro and in vivo studies (8-11). Because the mRNA expression level of dCK was related to its enzyme activity and also its protein levels (11), a positive correlation was expected to be revealed in this study. CDA enzyme activity has been thus far negatively reported regarding its association with gemcitabine sensitivity. In this study, however, neither of these genes was found to correlate with gemcitabine sensitivity.

RR consists of two subunits: M1 and M2. Ribonucleotide reductase enzymatic activity is modulated by levels of its M2 subunit (RRM2) (12). A p53-dependent gene, p53R2, has been found to encode a ribonucleotide reductase subunit (13). The p53R2 protein is similar to the M2 subunit and, through an association with the M1 subunit, forms an active ribonucleotide reductase heterodimer. Duxbury et al and Davidson et al respectively demonstrated that RRM1 and RRM2 were associated with gemcitabine resistance in pancreatic adenocarcinoma and non-small cell cancer cell lines $(14,15)$. In this study, however, with a variety of pancreatic cell lines, the expression of these genes was not positive for sensitivity to gemcitabine.

Transporter genes are essential for the efficacious action of any drug. Although transporter genes were not positively reported regarding 5-FU sensitivity, several genes such as hENT2, hCNT1 and hCNT3 have been well investigated for gemcitabine sensitivity (16-19). In this study, hENT1 was the only one gene in which we found a positive correlation with gemcitabine sensitivity. From the theoretical point of view, hENT1 is known to be a major gemcitabine transporter that is constitutively expressed in human pancreatic adenocarcinoma cells. For the next step of this study, we are planning on modulating the expression of this gene to examine whether it may change the $\mathrm{IC}_{50}$ of these pancreatic cell lines for gemcitabine. Another important approach is to investigate hENT1 mRNA expression in clinical samples and its correlation to the clinical effect. Recently, primary systemic treatment has become routine for cancer treatment, especially against pancreatic cancer for which the possibility of curative surgery is less than in other types of gastrointestinal tumors. It is interesting to examine the pathological effect after gemcitabine treatment and hENT1 expression in pancreatic tumors, although some modification may occur by the treatments.

We demonstrated that increased hENT1 expression is a potent determinant of gemcitabine sensitivity in pancreatic adenocarcinoma and biliary tract carcinoma cells. Further studies are needed to determine whether hENT1 would be useful as a predictive marker of sensitivity in the clinical setting. 


\section{References}

1. Burris HA III, Moore MJ, Andersen J, et al: Improvements in survival and clinical benefit with gemcitabine as first-line therapy for patients with advanced pancreas cancer: a randomized trial. J Clin Oncol 15: 2403-2413, 1997.

2. Huang P, Chubb S, Hertel LW, Grindey GB and Plunkett W: Action of 2',2'-difluorodeoxycytidine on DNA synthesis. Cancer Res 51: 6110-6117, 1991 .

3. Huang $P$ and Plunkett $W$ : Induction of apoptosis by gemcitabine. Semin Oncol 22: 19-25, 1995.

4. Plunkett W, Huang P and Searcy CE: Gemcitabine: preclinical pharmacology and mechanisms of action. Semin Oncol 23: 3-15, 1996.

5. Achiwa H, Oguri T, Sato S, Maeda H, Niimi T and Ueda R: Determinants of sensitivity and resistance to gemcitabine: the roles of human equilibrative nucleoside transporter 1 and deoxycytidine kinase in non-small cell lung cancer. Cancer Sci 95: 753-757, 2004

6. Ruiz van Haperen VW, Veerman G, Eriksson S, Stegmann AP and Peters GJ: Induction of resistance to 2',2'-difluorodeoxycytidine in the human ovarian cancer cell line A2780. Semin Oncol 22: 35-41, 1995 .

7. Bergman AM, Pinedo HM and Peters GJ: Determinants of resistance to 2',2'-difluorodeoxycytidine (gemcitabine). Drug Resist Updat 5: 19-33, 2002.

8. Ruiz van Haperen VW, Veerman G, Eriksson S, et al: Development and molecular characterization of a 2',2'-difluorodeoxycytidine-resistant variant of the human ovarian carcinoma cell line A2780. Cancer Res 54: 4138-4143, 1994.

9. Goan YG, Zhou B, Hu E, Mi S and Yen Y: Overexpression of ribonucleotide reductase as a mechanism of resistance to 2,2difluorodeoxycytidine in the human KB cancer cell line. Cancer Res 59: 4204-4207, 1999.

10. Van der Wilt CL, Kroep JR, Bergman AM, et al: The role of deoxycytidine kinase in gemcitabine cytotoxicity. Adv Exp Med Biol 486: 287-290, 2000.
11. Kroep JR, Loves WJ, van der Wilt CL, et al: Pretreatment deoxycytidine kinase levels predict in vivo gemcitabine sensitivity. Mol Cancer Ther 1: 371-376, 2002.

12. Eriksson S, Gudas LJ, Clift SM, Caras IW, Ullman B and Martin DW Jr: Evidence for genetically independent allosteric regulatory domains of the protein M1 subunit of mouse ribonucleotide reductase. J Biol Chem 256: 10193-10197, 1981.

13. Tanaka H, Arakawa H, Yamaguchi T, et al: A ribonucleotide reductase gene involved in a p53-dependent cell-cycle checkpoint for DNA damage. Nature 404: 42-49, 2000.

14. Duxbury MS, Ito $H$, Zinner MJ, Ashley SW and Whang EE: RNA interference targeting the M2 subunit of ribonucleotide reductase enhances pancreatic adenocarcinoma chemosensitivity to gemcitabine. Oncogene 23: 1539-1548, 2004.

15. Davidson JD, Ma L, Flagella M, Geeganage S, Gelbert LM and Slapak CA: An increase in the expression of ribonucleotide reductase large subunit 1 is associated with gemcitabine resistance in non-small cell lung cancer cell lines. Cancer Res 64: 3761-3766, 2004.

16. Mackey JR, Yao SY, Smith KM, et al: Gemcitabine transport in xenopus oocytes expressing recombinant plasma membrane mammalian nucleoside transporters. J Natl Cancer Inst 91: 1876-1881, 1999.

17. Ritzel MW, Ng AM, Yao SY, et al: Recent molecular advances in studies of the concentrative $\mathrm{Na}^{+}$-dependent nucleoside transporter (CNT) family: identification and characterization of novel human and mouse proteins (hCNT3 and mCNT3) broadly selective for purine and pyrimidine nucleosides (system cib). Mol Membr Biol 18: 65-72, 2001.

18. Mackey JR, Mani RS, Selner M, et al: Functional nucleoside transporters are required for gemcitabine influx and manifestation of toxicity in cancer cell lines. Cancer Res 58: 4349-4357, 1998.

19. Garcia-Manteiga J, Molina-Arcas M, Casado FJ, Mazo A and Pastor-Anglada M: Nucleoside transporter profiles in human pancreatic cancer cells: role of hCNT1 in 2',2'-difluorodeoxycytidine-induced cytotoxicity. Clin Cancer Res 9: 5000-5008, 2003. 